ROCZNIKI HUMANISTYCZNE

Tom LXVI, zeszyt 8 - 2019

DOI: http://dx.doi.org/10.18290/rh.2019.67.8-5

IWONA PIECHNIK

\title{
NOMS DE FAMILLE ISSUS DE L'ARTISANAT EN FRANCE ET EN POLOGNE
}

\author{
SURNAMES FROM ARTISAN NAMES IN FRANCE AND IN POLAND
}

\begin{abstract}
The article analyses surnames originating from artisan names in France and in Poland. It presents their origins (including foreign influences), types and word formation. We can see, among other things, that the French surnames are shorter, but have many dialectal variants, while the Polish surnames are longer and have a richer derivation. The article also focuses on demographic statistics of such surnames in both countries: the blacksmith as an etymon is the most popular. In the top 50, there are also in France: baker, miller and mason; while in Poland: tailor and shoemaker.
\end{abstract}

Key words: family names; surnames; patronyms; handicraft; artisan.

Les plus anciens noms de famille issus des domaines de l'artisanat en France et en Pologne remontent au Moyen Âge, donc à l'époque où le système féodal se renforçait et les villes commençaient à se développer, en nourrissant surtout les ambitions des nobles de construire leurs demeures seigneuriales, et des gens de petits métiers venaient s'installer tout autour naturellement. Dans des bourgs, c'est-à-dire dans de gros villages où se tenaient ordinairement des marchés, les bourgeois bénéficiaient d'un statut privilégié et développaient le commerce et la conjoncture de la manufacture, donc il y avait aussi beaucoup de travail pour différents métiers. C'est justement dans les bourgs et les villes que l'artisanat se développait le mieux, en

Dr hab. IWONA PIECHNIK - maître de conférences en linguistique à l'Institut de Philologie Romane de l'Université Jagellonne de Cracovie, Pologne. Elle s'intéresse à la linguistique historique et typologique, à la sociolinguistique et aux études historico-culturelles, ainsi qu'à l'évolution de la communication à travers les médias et à l'essor des technologies électroniques du point de l'usage de la langue ; courriel : iwona.piechnik@uj.edu.pl; ORCID : 0000-0003-3235-8122. 
se subdivisant en diverses spécialités (p.ex. couturier, mercier, drapier, boutonnier, chapelier, coiffeur, tapissier, serrurier, poêlier, ferblantier, etc.). Cependant à l'époque, encore env. $80 \%$ de toute la population travaillaient dans l'agriculture, donc beaucoup de gens de petits métiers non-artisanaux restaient toujours à la campagne (p.ex. gardiens d'animaux, moissonneurs, faucheurs, meuniers, etc.). Bien sûr, certains métiers pouvaient être ruraux et urbains à la fois : forgeron, charron, charpentier, menuisier, tonnelier, maçon, tisserand, tanneur, mégissier, cordonnier, chandelier, potier, miroitier, orfèvre, lormier, fondeur, mouleur, etc.

Le même métier était souvent exercé de père en fils (ou beau-fils), et ainsi pendant des générations ${ }^{2}$, par conséquent, les descendants héritaient non seulement de l'atelier et des outils, mais aussi d'un surnom issu du métier, de son atelier, de ses outils ou de ses produits. Ainsi, par le processus de proprialisation, le nom commun devenait le nom propre. Ensuite, il subissait d'autres procédés morphologiques, surtout la dérivation suffixale.

Les premiers documents français où les noms de métiers apparaissent, datent du XIII ${ }^{\mathrm{e}}$ s. : d'abord, nous avons le Livre des métiers (ou exactement Les Établissements des métiers de Paris) d'Étienne Boileau (prévôt de Paris) d'env. 1268. C'est le $1^{\text {er }}$ grand recueil de règlements sur les métiers (surtout grâce aux corporations). Mais du point de vue de l'onomastique, le plus important est le Rôle de la taille ${ }^{3}$ de 1292, rédigé à Paris, sous Philippe le Bel (voir Géraud 1837). Ce document énumère les contribuables, en donnant leur prénom et leur métier, p.ex. Symonnet, le cervoisier; ou leur prénom, le nom de leur vill(ag)e natal(e) et leur métier, p.ex. Nicolas de Compigne, cordoanier; ou bien leur prénom, leur surnom et leur métier, p.ex. Guillaume la Chièvre, péletier ou Guillaume le sage, mesgeicier ${ }^{4}$ ou bien Ameline, la couturière, la vielle ${ }^{5}$. Assez souvent, le prénom est suivi de deux termes professionnels, p.ex. : Jehan le Péletier, ferpier ${ }^{6}$ ou Henri le fèvre, serreurier. L'orthographe est instable, parfois on voit la majuscule, parfois la minuscule, donc le nom et le surnom se confondent. Rarement on peut y voir une information claire comme : Richart, le maçon, dit le boudinier. En somme, on ne peut pas discerner si le nom cité est issu de la profession exercée par ce

\footnotetext{
2 Justement, les changements de métiers n'étaient plus fréquents que dans les grandes villes (Dauzat 1949 : 163). En outre, on pouvait cumuler deux ou trois métiers chez une personne.

${ }^{3}$ Il s'agit de l'impôt annuel et permanent, levé presque uniquement sur les gens de métiers.

${ }^{4} \mathrm{C}$-à-d le mégissier.

${ }^{5}$ Il s'agit plutôt d'une vieille femme, et non pas de la vielleuse (cf. Kremer 1997 : 331).

${ }^{6} \mathrm{C}$-à-d le fripier.
} 
personnage ou par ses ancêtres, donc il faut «être très circonspect lorsque l'on rencontre, à date ancienne, un nom de baptême suivi d'un nom de métier» (Lebel 1959 : 91).

La valeur de ce document est grande aussi parce qu'on peut y voir des noms de métiers qui ne sont plus en usage, p.ex. mazelinnier, madelinnier 'ouvrier qui fabriquait "des coupes à boire réservées pour l'usage des grands et des riches ${ }^{, 7}$ (Géraud 1837 : 522) ou maignen "chaudronnier ambulant, rétameur', ainsi que les anciens noms de métiers que nous connaissons, p.ex. mire/meire 'médecin' et mirgesse/meiresse 'femme qui pratiquait la médecine'.

Certains sobriquets étaient passagers, mais d'autres restaient et devenaient héréditaires. Enfin, les devoirs administratifs ont imposé la notation des noms de famille. En France, c'est l'ordonnance de Villers-Cotterêts de 1539 qui instaure le registre civil de tous les sujets ${ }^{10}$, tenu par les paroisses et où le nom accompagne le prénom obligatoirement ${ }^{11}$.

En Pologne avec un long démembrement territorial de principautés, l'État commun se formait plus tard. Le développement social et culturel, y compris celui des villes, se faisait plus lentement. Enfin, au XIII ${ }^{\mathrm{e}}$ s., des colonisateurs allemands et hollandais sont arrivés, encouragés par des princes polonais. Ils ont apporté une nouvelle culture commerciale et de nouveaux métiers (ou plutôt des spécialisations), ainsi que les nouveaux droits de la fonctionnalité des villes (droits de Magdebourg) ce qui a favorisé l'accélération urbaine du pays (Kaleta 1998 : 27-28), ainsi que, sans doute, la valorisation du travail

\footnotetext{
${ }^{7}$ Le nom vient sans doute du madre «bois veiné utilisé au Moyen Âge pour fabriquer des vases à boire ", si bien que madre a pris le sens de 'vase à boire' et a fini par «désigner les pierres précieuses (agate, onyx) dont on faisait les coupes » (TLFi). Dans l'INSEE, on trouve Madre et Lemadre qui peuvent être liés à ce métier, mais pas de mazelinnier ni madelinnier.

${ }^{8}$ L'étymologie de ce mot est incertaine, mais le FEW (VI/1 : 2020) propose lat. *manianus 'Handarbeiter' < lat. manus 'main'. C'est probable vu qu'en italien nous avons : magnano 'Artigiano che esegue minuti lavori in ferro, come chiavi, serrature, maniglie, ecc.; con sign. più generico, fabbro' (cf. Treccani). Les noms de famille français issus de ce nom sont: Magni(e)n, Magnin, Maigneu, Maignan, Meigni(e)n, Magnan(t), Ma(i)gnet, Ma(i)gnot, Lema(i)gnan, Lema(i)gnen(t) (cf. Dauzat, $1951: 404$ et INSEE).

${ }^{9}$ Du lat. medicus, « avec évolution particulière du $d$ en $r$ caractéristique de mots d'infl. sav. » (TLFi)

${ }^{10}$ Plus tôt, on n'enregistrait que les noms des nobles (surtout pour des raisons armoriales) qui utilisaient les prénoms (souvent plusieurs) accompagnés de la fameuse particule de suivie du nom de leur localité d'origine. Ensuite, même les nobles avaient souvent des noms de famille issus de noms communs, auxquels on ajoutait un titre de noblesse accompagné de la préposition de suivie du nom de leur localité d'origine.

${ }^{11}$ Bien sûr, leur orthographe variait pour des raisons historiques (la norme orthographique vacillait et le clergé des paroisses notait les noms d'après une prononciation approximative et une vague connaissance des règles de la graphie usuelle).
} 
artisanal. C'est alors que les premières corporations des métiers s'organisent dans le Royaume de Pologne.

Quant aux patronymes polonais issus des métiers, on les voit tard, tout comme les noms de famille en général. En fait, la tradition des noms de famille émerge dès le $\mathrm{XIV}^{\mathrm{e}} \mathrm{s}$., mais ne prend d'envergure qu'au $\mathrm{XVI}^{\mathrm{e}} \mathrm{s}$. Cependant les premiers noms cités dans les documents sont ceux des nobles ${ }^{12}$, ensuite ceux de la riche bourgeoisie (d'abord chez celle d'origine allemande) ${ }^{13}$. Les surnoms des roturiers n'étaient pratiquement pas héréditaires jusqu'au XVIII ${ }^{\mathrm{e}}$ s. (Rymut 1991 : 21). ${ }^{14}$ Cependant, déjà au XVI ${ }^{\mathrm{e}}$ s., on peut trouver des documents urbains avec des prénoms plus ou moins latinisés et accompagnés de noms de métiers, écrits en latin (p.ex. Paulus faber, Stanislaus Sartor, Mathias brascator, Mathias pistor) ou en polonais (p.ex. Michael Kowal, Mathis krawiecz, Marcin piekarz, Stephanus Kaletnik) ; pourtant, on doit considérer ces termes plutôt comme un élément supplémentaire de la description des gens que leurs noms héréditaires (Rudnicka-Fira 2004 : 55). On peut y voir aussi une notation vacillante quant aux lettres minuscules et majuscules.

Une telle «description» des artisans en Pologne au XVI ${ }^{\mathrm{e}}$ s. nous rappelle celle du Rôle de la taille de 1292 en France. Mais on voit bien le décalage de presque trois siècles entre les deux pays dans ce domaine et dans l'enregistrement des anthroponymes en général.

\section{NOMS ARTISANAUX FRANÇAIS}

Il s'agit des noms de famille d'origine latine et populaire, sans être empruntés tardivement au latin «savant». Nous ne prenons pas en compte les noms issus d'autres langues romanes. Nous ne citons que quelques exemples pour chaque type.

\footnotetext{
${ }^{12} \mathrm{Au} \mathrm{XII}{ }^{\mathrm{e}}$ s., c'était une sorte de description de l'origine, donc on appliquait la préposition pol. $z$ ou lat. $d e+$ localité natale. Depuis le XIII ${ }^{\mathrm{e}} \mathrm{s}$. on commence à surnommer les gens par des noms communs. C'est alors aussi que les surnoms "adjectivaux » en -ski (désignant la provenance) apparaissent, mais ils sont rares jusqu'au $\mathrm{XVI}^{\mathrm{e}} \mathrm{s}$. Curieusement, à cette époque, les roturiers ont commencé à utiliser cette terminaison aussi (Kaleta $1998: 27,31-33$ ).

${ }^{13}$ N'oublions pas que les documents écrits conservés sont tardifs et en latin, donc les premiers noms cités sont rares aussi (le premier document avec quelques prénoms polonais cités dans le texte latin est la Bulle de Gniezno de 1136). Longtemps les scribes avaient aussi du mal à noter leur prononciation avec l'alphabet latin.

${ }^{14}$ Et leur codification se faisait jusqu'au XIX ${ }^{\mathrm{e}}$ s., différemment sur les terrains polonais partagés entre la Russie, la Prusse et l'Autriche (Rymut 1991 : 25).
} 


\section{I.1. NOMS ISSUS DU LATIN, DONT L’ÉTYMON A DISPARU DANS LA LANGUE FRANÇAISE}

lat. faber ${ }^{15}$ 'forgeron' : Fabre (sud-est), Faré (Roussillon), Fèvre, Lefèvre (langue d'oïl), Faivre (est d'oïl), Feuvre, Lefeuvre (ouest d'oïl), Fèbre, Febreau, Feubre, Lefeubre, Lefebre (Poitou), Favre (franco-provençal), Faur(e) (nord de la langue d'oc), Haur(e), Hauresse, Haurillon, Haury (Gascogne) - ce métier était le plus répandu au Moyen Âge, donc le surnom s'est popularisé, pour ensuite devenir un nom de famille ${ }^{16}$. Cela n'empêche pas que, comme Albert Dauzat l'explique, «le type faber a disparu devant maréchal, voire forgeron, dans une grande partie de la France; mais quand les noms de famille se sont formés, il était encore d'un usage général et courant (sauf réserves pour l'Ouest)» (Dauzat 1951 : 320). Selon Dauzat, le forgeron ${ }^{17}$ ne l'a supplanté qu'au XVI ${ }^{\mathrm{e}} \mathrm{s}$. ;

lat. sutor 'cordonnier' : Sueur, Lesueur, Sudre, Sudraud, Sudour, Sutter(lin), Suttern;

lat. sartor 'ravaudeur, raccommodeur' > Sartor, Sart(h)ou, Sartore(l), Sartre(s), Sa(s)tre, Sartieaux, Sarton, Sartori(u)s, Sartory;

lat. macellarius 'boucher': Metz(e)ler, Metzger, formé en Alsace-Lorraine (Dauzat $1951: 432$ ). Cet étymon a laissé sa trace aussi dans l'ancien mot fr. maiselier 'boucher' 18 .

\section{I.2. NOMS D’ORIGINE LATINE, NÉS SUR LA BASE DÉJÀ FRANÇAISE, DONT L'ÉTYMOLOGIE EST DIFFICILEMENT RECONNAISSABLE}

Boucher, Boucherand, Boucheras, Boucherat, (La) Bouchère, Boucheret, Boucherez, Boucherie, Boucheron, Boucherot, dans le Nord : Bouquier = boucher, créé à la fin du XII ${ }^{\mathrm{e}}$ s., avec une teinte péjorative: 'celui qui abat les boucs et vend cette viande', mais ce nom s'est répandu, en neutralisant sa signification et en éliminant anc.fr. maiselier ;

\footnotetext{
${ }^{15} \mathrm{La}$ seule trace de cet étymon dans la langue française d'aujourd'hui ne reste que dans l'orfèvre qui est un remaniement du lat. aurifex > lat. tard. aurifaber.

${ }^{16}$ Actuellement il est peut-être l'un des plus étudiés parmi les noms de famille en France. Nous donnons sa répartition géographique selon Dauzat (1951: 320-322, voir aussi ALF). Dauzat dit aussi qu'il y a des hameaux Chez Favre ou Chez Faure.

${ }^{17}$ Le forgeron est issu de l'évolution du lat. fabrica 'atelier' $>$ fr. forge $\left(\mathrm{XII}^{\mathrm{e}} \mathrm{s}\right.$. $)>$ forgeur $\left(\mathrm{XIII}^{\mathrm{e}}\right.$ s.) > fr. forgeron $\left(\mathrm{XVI}^{\mathrm{e}} \mathrm{s}\right.$.). Nous citons les dates des premières attestations d'après le TLFi.

${ }^{18} \mathrm{Il}$ y a aussi des noms de famille : Maisel, Maizel, Mézel, issus du lat. macellum 'marché aux viandes' (< lat. macello,-are 'tuer des animaux').
} 
Boulang(i)er, Boulangez, Boulangée, Boulangey, Boulangeot, Bouleng(i)er, Boulange, Boulenge, Boulenc, Boulenguer, Boulenguez, Le Boulanger, Le Boulenger, Leboulanger, Leboulenger $<$ 'celui qui fabrique le pain en boule', le boulanger a éliminé le fournier ;

Boursier 'fabricant de bourses, ${ }^{19}<$ fr. bourse 'petit sac' $<$ lat. bursa 'cuir' ;

Chapu(i)s, Chaput $<$ fr. (dial.) chapus/chaput 'billot de bois sur lequel on équarrissait les ardoises et les douves des tonneaux' < fr. chapu(i)ser 'fendre du bois en petits morceaux' < lat. *capputiare < lat. *cappare (cf. FEW II : 279-283 et TLFi),

(Le) Charpentier, Lecharpentier, Charpenteau, Charpentreau, Charpentron, Carpentras, De/Le Carpentier, Lecarpentier, Decarpent(e)rie(s), Descarpent(e)rie(s), Descarpentry < lat. *carpentarius 'charron' < lat. carpentum 'char à deux roues';

Cordonnier, Lecordonnier - d'abord 'marchand de cordons', ensuite 'marchand de chaussures et fabricant de chaussures en cuir de Cordoue' $(<$ cordon + Cordoue, cf. infra);

Courvoisier, Corv(o)isier, Courvasier, Crouvoisier, Corvisy, Crouvezier, Crevisier, Corvisart, Lecorvaisier, Le Corvoisier, Le Corvasier "cordonnier' < anc. fr. corvisier, corvixier, corbesier, corbusier, etc. (cf. FEW II : 1182) < anc. fr. corvois $\sim \operatorname{cordo}(u)$ an $\sim$ cordowan 'cuir de Cordoue'20 (cf. ibidem et Godefroy II : 300, 321, 338) ;

Lor(re)mier ('fabricant de lorains, de harnais, de freins') $<$ anc. fr. lorain 'courroies de cuir du harnais' < lat. lorum 'cuir, courroie, rêne' (cf. Godefroy V : 31-33);

Patenôtre, Paternot(te) < fr. patenôtrier 'fabricant de patenôtres (chapelets)' $<$ lat. pater noster;

Meunier, Meusnier, Mu(s)nier, (Le) Mounier, Lem(o)unier, Mugnier, Dumunier, Mugneret, Mugnerot < anc. fr. mounier, munier $<$ lat. molinarius 'meunier';

Menuisier $<$ fr. menuiser $<$ fr. menu 'mince, petit' < lat. minutus ; ce mot apparaît au XIII' s. ; avant, un tel ouvrier de menus ouvrages en bois était appelé chapuis.

\footnotetext{
${ }^{19}$ Ce mot a changé sémantiquement. Il ne s'agit donc pas du boursier au sens actuel qui découle de l'évolution sémantique du mot bourse ('petit sac pour porter de l'argent' > 'réserves d'argent').

${ }^{20}$ Le fameux cuir s'appelle en espagnol cordobán (ou guadameci $\sim$ guadamecil - mot d'origine arabe), d'où les formes d'autres langues, p.ex. ang. cordovan, cordwain, ou pol. kurdyban.
} 


\section{I.3. NOMS D’ORIGINE LATINE, NÉS SUR LA BASE DÉJÀ FRANÇAISE, FACILEMENT RECONNAISSABLES}

Nous pouvons les diviser selon le type du mot ayant servi de base :

- l'outil ou l'objet du travail: (Le) Bourre, Bourrel(l)ier, Bourlier, Bourliaud, Lebourlier = bourrelier ; Fourn(n), Le Fourn(is), Fourneau, Fournaud, Fourno(t), Fourneret, Fourneron, Fournerot, (Le) Fournier, Lefournier, Fournière, Lafournière, Fournerie, Fournery, Dufour, Dufournier, Dufourn(e)au(d), Dufournet = boulanger; Marteau, Martel(l), Martelat, Martel(l)et, Martelot, Martel(l)eur, Martellier, Martelon, Lemarteleur, etc. $=$ forgeron (ou charpentier ?), Mégis(sier) = mégissier ; Savat(t)e, Savat(t)ier, Savaton, Savetier, dans le Midi : Sabat(t)ier, Sabathier, Sabaton, Sabatou, Sabathe, Sabat $(t) e=$ savetier $^{21}$;

- le produit du travail : Chapeau(x), Chapelier, Lechapelier, Le Chapellier, dans le Midi : Capel(lier), Capellot = chapelier, celui qui fait des chapeaux ; C(h)arron, Char(re)tier, Charreton, Lechartier, Le Chartier, Charronneau, Cherrier, dans le Midi : Carreton, Carron(nier), Cartier = charron ; Farine, Far(i)nier, Farineau(x), Farinet , Farinot(tte) $=$ meunier ; Pain, Pan(n)et, Pan(n)etier, Panneton = boulanger (ou marchand de pain) ; Roue(t), Rode(au), Ro(d)dier, Rodié = fabricant de roues, charron ; Toil(l)ier, Toiliez, Toill(i)on, Tel(l)ier, Tel(h)iez, Teleron, Let(h)ellier, Lethelliez, Leteillier $=$ toilier $(<\text { lat. tela 'toile' })^{22}$;

- l'endroit du travail: (La) Forge, Def(f)orge(s), Delaforge, Deleforge, Delforge(s), Desforge(s), Forgeais, Forgeard, Forgeas, Forgeat, Forgeau(d), Forgeois, Forgeon, Forgeot, Forgeoux, Forgerais, Forgereau, Forgeret, Forgeron(t), Forgerou, Forges, Forget(te), Forgez, Laforge(rie), Leforgeais $=$ forgeron $(<$ fr. forge $<$ lat. fabrica $) ;$ Moulin(s), Moulinard, Moulinas, Moulinat, Moulineau, Moulinec, Moulines, Moulinet, Moulinier, Moulinot, Moulinou(x), Lemoulinier, Dumoulin, Lamouline, dans le Midi : Molinier, Molinet, Molinot, Molineau, Molins = meunier ;

- de l'action faite au travail (verbe): coudre/couture: (Le) Couturier, Lecouturier, Lacouture, La Couturière, Decouture, Descouture(s), Couture(s), Couture(au(x)), Couturet, Couturieux, Couturiaux, Couturie,

\footnotetext{
${ }^{21}$ On peut y citer aussi p.ex. barbe ou bien serrure avec ses équivalents occitans sarrail, sarralha, etc.

${ }^{22}$ Ce type est très répandu, on peut y citer encore p.ex. arc, baril, bouton, chandelle, chaudron, chaussette, clou, coiffe, corde, coupe, drap, goudron, lampe, lanterne, moutarde, parchemin, parement, peigne, plâtre, pot, savon, selle, soulier, tonneau, van, etc.
} 
Coudurier, Coudures; couper: Coupeur, Lecoupeur ${ }^{23}$; tailler: Letaill(i)eur, Letailleux, Destailleur, Destailleurs, Tailleu(x), Tailleur, Tailland, Taillandie(r), De/Le Taillandier, Letaillandier 'couturier'"24; tisser: Tissier, Letissier, Tissandier, Tixier, Tisserand, Letisserand, Texier, Letexier, Tesseyre, Tessier, Lete(i)ssier, Teissèdre, etc. ${ }^{25}$

\section{I.4. NOMS D'ORIGINE GERMANIQUE, NÉS SUR LA BASE DÉJÀ FRANÇAISE}

Il s'agit des noms d'origine germanique, bien implantés dans l'histoire de la France et du français. Ils sont donc plutôt liés à l'arrivée de peuples germaniques au $\mathrm{V}^{\mathrm{e}}$ s., surtout à l'installation des Francs et à l'instauration de leur empire. On voit la plus grande popularité de deux noms de métiers :

maréchal (< germ. *marhskalk) 'domestique chargé de soigner les chevaux', puis, en tant que maréchal-ferrant 'artisan dont le métier est de ferrer les chevaux et les animaux de trait' > Maréchal(le), Maréchau(d), Maréchaux, Marécheau, Mareschal, Mareschal, Marschalk, Marschall, Mar(i)chal, Maricot, Maricau, Mariscal, Maresco, Marisco, Marischa(e)l, Marichaud, Lemar(é)chal, etc.

maçon (< francique *makjo) : Maçon(nerie), Masson ${ }^{26}$, Masson(n)et, Masson-

nat, Massonnaud, Massonneau, Masson(n)ot, Massenat, Massenet,

Massenez, Massenot, Lemasson, Le Masson, Lemaçon, etc.

Dans cette catégorie, on peut mettre aussi des noms de métiers, dont la base est un mot d'origine germanique, désignant un produit ou un objet du travail (et non pas un métier), p.ex. heaume $\left(<\right.$ frq. ${ }^{*}$ helm $\left.{ }^{27}\right):$ Heau(l)me et son dérivé français heaumier caché dans: Laumier, Laumière, Leaumier,

${ }^{23}$ Dans la briqueterie, cet ouvrier coupait et façonnait la terre pour les briques, mais il y a aussi eu d'autres coupeurs : de cheveux, de hanses (chez les épingliers), de poils (c-à-d cardeurs), de racines (herboristes), de têtes (chez les épingliers), de tronçons (rogneurs) (Franklin $1906: 220$ ).

${ }^{24}$ Le mot tailleur était appliqué à tailler aussi d'autres objets que le vêtement (p.ex. tailleur de pierres), donc le couturier, en tant que terme plus précis, l'a supplanté.

${ }^{25}$ On peut y citer aussi, p.ex. les verbes fouler, fondre, mouler, tourner, ou l'action de teindre : teinture, etc.

${ }^{26} \mathrm{Ce}$ nom peut venir aussi du prénom Thomas, particulièrement de son hypocoristique Thomasson. Il est possible que les deux formes se soient confondues, voir Dauzat (1949 : 126-127, 168 ; 1951: 168).

${ }^{27}$ Cet étymon se cache aussi dans le prénom Guillaume $<$ Wilhelm (will 'volonté' + helm 'casque, heaume'). 
Haumier, etc. Il en va de même avec gant (< frq. *want) : Gant, Gant(h)ier, Gantiez, Gantillon ou haubert ( $<$ frq. *halsberg) : Hauber(t), Haubertin, etc.

\section{I.5. NOMS ISSUS DE MOTS D’ORIGINE GAULOISE}

Ils sont peu nombreux, dérivés de quelques étymons gaulois, souvent passés par l'intermédiaire du latin : battre : Battandier, Batteur $(<$ batteur de fer/d'or, etc.), (Le) Batteux, Lebatteur, Lebatteux; braies : Braie, Brayer, Brayère; cervoise: Cervoise (peut-être comme 'brasseur'); claie : Clay, Claye(s), Clayer, Clayet(te), Clayeux, Clayon; tan(ner): Tanneur, Letanneur, Tanneau, Tanneux, Tanret, etc.

\section{I.6. NOMS COMPOSÉS}

Ils sont assez rares parmi les noms de famille en général, et encore moins parmi les noms issus des métiers. Il s'agit surtout des "métaphores diverses et formations ironiques " "dont la valeur est plus ou moins obscure » (Dauzat 1949 : 206).

- boulanger : Beaupain, Blan(c)pain, Bompain, Bonpain, Dupain, Feuillepain, Lepain, Painblanc, Painchaud, Painchault, Painchaux, Paindavoine, Paincuit, Paindépice, Paindorge, Painlevé, Painsec, Paintendre, Petitpain, Richepain, Malcuit, Maucuit, Maupain, Monpain, Taillepain, Tornemiche, Tranchepain, Galmiche, Fourneuf, Fourneuve ${ }^{28}$

- meunier : Mouillefarine, Gastefarine, Poncegrain, Échallegrain, Sauvegrain, Cassegrain, Gâteblé, Tournesac, Beaumunier, Moulinjeune, Moulinneuf, Touchemoulin, Troismoulins

- forgeron : Taillefer, Maillefer, et son marteau : Bonmartel, Commarteau, Demarteau, Malmartel, et sa forge : Forgemont, Forgeneuf, Forgeneuve, etc.

Parmi d'autres métiers, la composition est plus rare: pâtissier: Brisetarte; boucher: Beauboucher, Beaubouchez; serrurier: Malhape ('mauvais crochet'); cordonnier: Chaussepied, Chauffepied, Tail(le)pied, Tailpied, Malchausse, Mauchaussat; couturier: Taillecourt, Taillefesse;

\footnotetext{
${ }^{28}$ Parfois il est difficile de discerner à qui un ancien surnom se réfère. Dauzat dit que les sobriquets comme Brisemiche, Brisepain, Cassemiche, Tu(e)p(a)in, Machetourle expriment plutôt l'appétit des consommateurs (Dauzat 1949 : 206).
} 
gantier: Taillemite ; menuisier: Taillebois ; maçon : Malmasson ; potier : Malpot, orfèvre : Lorfeuvre, Lorfèvre, etc.

\section{I.7. NOMS ISSUS DE LANGUES RÉGIONALES NON-ROMANES ${ }^{29}$}

- allemands : boulanger : Backer, Becker, meunier : Muhler, Mühler, Muller, Müller, Muhlmann ${ }^{30}$, cordonnier: Choumaker, Schoumacher, Sch(o)uma(c)ker, Sch(o)uman(n); forgeron: Schmid(t), Schmider, Schmidtke, Schmied(t), Schmieder(er), Schmit(d), Schmith, Schmitt(e)(r), Schmittler, Schmittlin, Schmitz(er), aussi Hammer 'marteau', Hammerer, Hammerschmidt, Hammerschmitt, Huf(f) schmid(t), Huf(f) schmitt ${ }^{31}$, Kleinschmidt $t^{32}$, etc.

- bretons : forgeron : (Le) Goff, Legof(f), Le Gô, Le Goffe, Legoffe, diminutifs : (Le) Goffic, Goïc, Le Goic, (Le) Govic, (Le) Gouic, composés : Gobian, Gobien, Gaubian, Goel, Gouel, Gou(v)ello, etc. (voir beaucoup plus de patronymes de métiers dans Abalain 2004 : 242-244).

- basques : arotz 'forgeron; charpentier' > Arocena, Arozamena, Arostegui, Arosteguy; arguin 'tailleur de pierre' : Arguindeguy, Harguindeguy 'chez le tailleur de pierre, maison du tailleur de pierre'. Il y a aussi un nom dont la forme fait indirectement penser à la forge : Olazábal, Olazabal 'cabane large', traditionnellement entendue comme 'forge' (cf. Michelena 1973, Alonso 2018, EAV).

La tradition des noms de famille issus de métiers est forte dans les régions germanophones (Alsace et Lorraine) ${ }^{33}$ et en Bretagne : ces patronymes y sont nombreux avec une riche morphologie. Par contre, parmi les noms de famille d'origine basque, les métiers sont extrêmement rares : ici le plus souvent, les patronymes évoquent la forme de la maison ou sa location ou bien des éléments du paysage.

\footnotetext{
${ }^{29}$ Nous citons ici uniquement des exemples trouvés dans le recensement actuel de l'INSEE, sans tenir compte de langues d'immigrés.

${ }^{30} \mathrm{Il}$ y a aussi beaucoup de noms composés avec le mot all. Mühle 'moulin': Muhl(en), Muhl(e)(n)bach, Muhlberger, Muhlemann, Muhlematter, Muhle(n)thaler, Muhlhauser, Muhlheim, Muhlmeyer, Muhlstein, avec ou sans le tréma sur le $u$.

${ }^{31}$ L'all. Huffeisen est le fer à cheval (Huf 'sabot' + Eisen 'fer').

${ }^{32}$ On peut y citer aussi des métiers de la ferblanterie, p.ex. : Kopferschmitt, Kupperschmitt (Kupfer 'cuivre'), Messerschmitt (Messer 'couteau') 'coutelier', Nagelschmitt (Nagel 'clou') 'cloutier', etc.

${ }^{33}$ Curieusement, en 1927, Henri Carrez a érit : « on constate avec étonnement que dans une ville comme Metz, où les corporations étaient abondantes et les artisans très nombreux, les métiers ont fourni peu de noms de famille, j'entends les noms de langue romane, car ceux d'origine allemande comme Becker, Müller, Choumacher sont très fréquents » (Carrez 1927 : 257-258).
} 


\section{I.8. STRUCTURE MORPHOLOGIQUE ET DIFFÉRENCES DIALECTALES}

La suffixation est plus fréquente que la composition. Deux suffixes désignant l'agent dominent :-(i)er et -eur, comme Dauzat le résume bien :

[...] en français central la plupart des noms de métiers sont pourvus du suffixe -ier (réduit plus tard à -er après $c h, g$ ). Le suffixe -eur (ancien -eeur, lat. -atorem) s'observe dans les dérivations verbales, quand le nom est tiré, non d'une denrée ou objet vendu ou fabriqué, mais d'une action [...]. Les noms appartenant à d'autres types sont rares : quelques mots traditionnels, latins (fèvre, marchand, queu[x]) ou germaniques (maréchal) et des isolés (cosson, chapuis). Le suffixe -on s'est ajouté à -ier (-er) dans porcheron, forgeron; -enc, altéré en -and, dans tisserand (Dauzat 1949 : 171).

On trouve aussi des suffixes diminutifs, surtout hypocoristiques (mais on ne doit pas exclure la manière non-affective d'appeler ainsi le fils d'un artisan ou son apprenti) : -et, -ot, -on, -eau. Dauzat y remarque curieusement aussi qu'ils

[...] affectent toujours le nom isolé, sans article, devenu surnom et dégagé de son origine: Fevrot, Tissot... doivent s'interpréter «le petit Fèvre», «le petit Tissier », et non « le petit forgeron », « le petit tisserand » (Dauzat $1949: 176$ ).

Dans de nombreux noms, on voit l'article défini, souvent uni avec le mot : «L'article se soude surtout aux mots courts et aux mots à initiale vocalique; dans les autres, son agglutination s'observe en Normandie et dans la région du Nord» (Dauzat 1951: 171).

S'il s'agit de la longueur des noms de famille français, Dauzat remarque que «le nom de métier polysyllabe peut être remplacé par le nom de l'objet vendu, symbolisant, par ellipse, le métier » (Dauzat 1949: 170). ${ }^{34}$

On observe aussi des différences régionales, issues de diverses lignes de développement vocalique et consonantique entre les domaines d'oül et d'oc, p.ex. [b] intervocalique dans le Midi et [v] dans le Nord, ou [a/a] dans le Midi et $[\mathrm{e} / \varepsilon / \mathrm{e}]$ dans le Nord, ou bien [o/o] dans le Midi et $[\mathrm{u}]$ dans le Nord, enfin la palatalisation de $[\mathrm{k}]$ et de $[\mathrm{g}]$ devant $[\mathrm{a} / \mathrm{a}]$ ne s'est pas effectuée en Normandie et en Picardie, etc.

\footnotetext{
${ }^{34}$ Même dans les noms de métiers français, on peut voir cette inclination pour la brièveté des formes : « les trisyllabes ont eu peu de succès, en dehors de métiers très usuels comme boulanger » (Dauzat 1951: 169).
} 
Les matronymes sont rares. Parmi les exemples cités, nous ne trouvons que : (La) Bouchère, Fournière, Lafournière, La Couturière, Laumière, Brayère.

De nombreux noms de métiers n'apparaissent pas parmi les noms de famille. Peut-être pour au moins trois raisons :

1) Certains noms de métiers étaient absorbés par d'autres, plus larges sémantiquement, p.ex. mortellier (qui maniait le mortier) $\rightarrow$ maçon, feutrier $\rightarrow$ chapelier, fourreur $\rightarrow$ pelletier, tabletier $\rightarrow$ menuisier, bijoutier et lapidaire $\rightarrow$ orfèvre, fondeur et mouleur $\rightarrow$ forgeron, épinglier, éperronier, émancheur, fermaillier (fabricant de fermoirs et d'agrafes), forcetier (fabricant de ciseaux), tréfilier $\rightarrow$ ferblantier, etc. (cf. aussi Dauzat $1949: 169$ ).

2) Certains noms de métiers « répandus aux XIII ${ }^{\mathrm{e}}-\mathrm{XIV}^{\mathrm{e}}$ siècles [...] devaient avoir des synonymes ou des sobriquets populaires » (Dauzat 1949 : 171).

3) Certains noms de métiers se sont créés quand les noms de famille en France se sont déjà fixés et stabilisés, au plus tard au XVI ${ }^{\mathrm{e}}$ s., p.ex. le perruquier qui « apparaît avec la mode des perruques à la fin du XVI ${ }^{\mathrm{e}} \mathrm{s}$, , coiffeur dans la seconde moitié du XVIII ${ }^{\mathrm{e}}$ s. » (Dauzat 1949: 169, note 1), tout comme les métiers encore plus tardifs, p.ex. l'aiguilleur, l'aviateur ${ }^{35}$, etc.

\section{NOMS ARTISANAUX POLONAIS}

\section{II.1. NOMS DÉRIVÉS}

Parmi les métiers populaires : Kowal 'forgeron' : Kowalski, Kowala(k), Kowalaszek, Kowalczak, Kowalczek, Kowalczuk, Kowalczyk(iewicz), Kowalczykowski, Kowalczyński, Kowalczys, Kowalec(ki), Kowalek, Kowalew(icz), Kowalewski, Kowalowic, Kowalik(owski), Kowalina, Kowaliszewski, Kowalkiewicz, Kowal(k)iński, Kowalkowski, Kowalnik, Kowalonek, Kowalówka, Kowaluk, et des formes dérivées du verbe kować 'battre (le fer)' : Kowar(a), Kowarczyk, Kowarnik, Kowarczyk, Kowarski, Kowarz(yk), Kowan(ek), Kowaniec, Kowański, Kowa(r)sz, Kowaszewicz, Kowat, Kowecki, Kowek, Kowiak, Kowic, Kowiel, Kowoń, Kowynia, Kowyniak, Kowacz ${ }^{36}$, Kowacki, Kowaczek, Kowaczka, Kowaczyk; Szewc 'cordonnier': Szewczyk, Szewcowicz, Szew-

\footnotetext{
${ }^{35}$ Mais on peut trouver Pilote ou Pilotin parmi les noms de famille en France, parce que jadis, le pilote servait dans la navigation des navires (cf. TLFi). La fréquence de tels patronymes est pourtant rare.

${ }^{36}$ Cette forme peut venir d'autres langues slaves (p.ex. serbo-croate kovač, bulg. ковач) ou du hongrois kovács (emprunté au slave). En Pologne on trouve aussi : Kowaczek, Kowaczyk, Kowaczew(ski), Kowaczik, etc.
} 
ców, Szewczak, Szewczenko, Szewczuk, Szewczykowicz, Szewczykowski, Szewczyński, Szw(i)ec, Szewiec(zek), Świec; Cieśla 'charpentier' : Cieślak(iewicz), Cieślakowski, Cieślan, Cieślar, Cieślarczyk, Cieślewicz, Cieślica, Cieślicz(ko), Cieślik(iewicz), Cieśluk(owski), Ciesiel(ka), Ciesielczak, Ciesielczuk, Ciesielczyk, Ciesiołka, Ciesiołkiewicz, Ciesiótka, Ciesiułka; Zdun 'poêlier, fumiste': Zdun(i)ak, Zdun(i)ek, Zduniewicz, Zdunik(owski), Zdunkiewicz, Zdunkowski, Zduń, Zduńczuk, Zduńczyk(owski), etc. ${ }^{37}$

Le plus souvent, les noms de famille sont liés au travail artisanal, issus d'outils, de produits (ou de leurs parties), de l'endroit et de l'action faite par l'artisan. Au début, appeler quelqu'un par un simple nom de son produit pouvait avoir une teinte plutôt péjorative (cf. Bubak 1986 : 157). Mais une riche dérivation polonaise (le plus souvent diminutive) a peut-être contribué à une rapide lexicalisation de tels noms :

- d'outils ou d'objets dont l'artisan se sert au travail : kopyto 'embauchoir' : Kopyt(k)o, Kopyt(a), Kopytarz, Kopytek, Kopytnik, Kopytow(ic), Kopyciak, Kopyciok) ; szydlo 'alène' : Szydło, Szydet, Szydłowicz, Szydelski, Szydlak, Szydlaczek, Szydlaczyk, Szydlik, Szydluk, Szydełko, Szydłowski ; żarna 'pilon': Żarna, Żarnawicz, Żarniewicz, Żarnoch, Żarnota, Żarnowiczka, Żorna, Żorniak, etc. ${ }^{38}$

- de produits du travail : kaleta/kalita 'sac, bourse' : Kaleta, Kalet(ka), Kaletnik, Kaleciak, Kaleciński, Kalit(k)a, Kalitowicz, Kaliciak, Kaliciński, Kaliciuk, Kaliczyński, Kalitta ; tagwa/lagiew (vieux-pol.) 'récipient pour les liquides' ${ }^{39}$ : Lagwa, Lagownik, Lagowniczek; rzeszoto 'van, tamis' : Rzeszot(czak), Rzeszotek, Rzeszotka, Rzeszotko, Rzeszotowski, Rzeszut(o), Rzeszutczak, Rzeszutek, Rzeszutko, etc. ${ }^{40}$

- d'une partie d'un produit : cholewa 'partie supérieure de la botte' : Cholewa(sz), Cholewi(a)k, Cholewczak, Cholewczuk, Cholewicz, Cholewiusz, Cholewjusz, Cholewo, Cholewski, Cholewczyński, Cholewiński, Cholew-

\footnotetext{
${ }^{37}$ On peut y citer aussi des dérivés d'autres mots : stolarz 'menuisier' ou tokarz 'tournier', parce que même si ces noms, munis du suffixe -arz '-ier' viennent d'autres bases : stót 'table' et toczyć 'tourner', les patronymes se sont créés sur la base des noms de ces métiers, p.ex. Stolarczyk, Stolarek, Stolarski, Stolarzewicz et Tokarczyk, Tokarzak, Tokarski, Tokarczuk, etc.

${ }^{38}$ On peut y citer aussi des dérivés d'autres mots : glina 'argile', młot(ek) 'marteau', pytel 'sac à farine', etc.

${ }^{39} \mathrm{Du}$ proto-slave *lagy/lagъve (cf. SEM). Comparez aussi lat. lagena 'bouteille, flacon, carafe ; gourde'.

${ }^{40}$ On peut y citer aussi des dérivés d'autres mots : cegła 'brique', chleb 'pain', garnek 'pot', grzebień 'peigne', guz(ik) 'bouton', gwóźdź 'clou', igła 'aiguille', kiełbasa 'saucisson', kociol 'chaudron', kosz 'panier', kożuch 'fourrure', krupa 'sorte de gruau', maka 'farine', mydlo 'savon', pancerz 'armure', powróz 'corde', stód 'malt', żelazo 'fer', etc.
} 
ziński; wątor 'jable du tonneau': Wątor(ek), Watora, Wątorczyk, Wątorowski, Watorski, Wontor ${ }^{41}$.

- de l'endroit du travail : kuźnia 'forge' : Kuźni(a)k, Kuźniar(z), Kuźnic(a), Kuźniczek, Kuźniec, Kuzniec ${ }^{42}$, Kuźniecow, Kuźniewicz, Kuźniewski, Kuznowicz, Kuźno, Kuźniarek, Kuźniarski, młyn 'moulin' : Młynarz 'meunier', Młynarzowic, Młynar(czak), Młynarczyk, Młynarczykowski, Młynarek, Młynarski, Młyniec, Młyni(u)k, Młynki, Młynkiewicz, Młynowski, Młyńczak, Młyńczyk, Mlenarski, Mynarz, Mynarski.

- de l'action faite au travail : krawać 'tailler, couper' : Krawiec 'tailleur, couturier', Krawczyk(iewicz), Krawczyński, Krawcow(icz), Krawczak, Krawczenko, Krawczewski, Krawczonek, Krawczuk, Krawczun, Krawczycki, Krawczykowski, Krawaczek, Krawalek, Krawania, Krawański, Krawar, Krawicz, Krawuczek, Krawul ; piec 'cuire' : Piekarz(yk) 'boulanger', Piekacz, Piekaj, Piekalkiewicz, Piekański, Piekara, Piekarczak, Piekarczyk, Piekarec, Piekarek, Piekarewicz, Piekarowicz, Piekarski, Piekarzewicz, Piekarzowicz, Piekorz, Piekosz, Piekoś, Piekta, Piekur, Piekurski, Piekus, Piekuś, Piekut(a), Piekutek, Piekutowski ${ }^{43}$.

Bien sûr, nous n'en avons montré que quelques exemples, mais on peut dire que les outils et les produits du travail sont plus populaires parmi les bases des noms que les parties de produits ou l'endroit du travail ou bien l'action faite par l'artisan.

Dans certains cas, on peut seulement présumer un lien d'un nom commun et du travail, sans être sûr des circonstances de l'attribution de ces noms, p.ex. quand quelqu'un s'appelle Bochen 'miche' (avec ses dérivés), on peut se demander s'il s'agit du descendant d'un boulanger ou de celui qui seulement apportait/vendait des miches ou bien qu'il aimait en manger.

\section{II.2. NOMS COMPOSÉS}

Ce type de noms de famille polonais est rare (Malec 2008 : 227), non seulement du point de vue du nombre de tels noms, mais aussi de la fréquence de leurs porteurs (entre parenthèses, nous donnons la somme des hommes et des femmes qui les portaient en 2018 en Pologne, selon le MC) :

\footnotetext{
${ }^{41}$ On peut y citer aussi des dérivés d'autres mots : otręba 'son (résidu de la mouture du blé)', pas 'ceinture', rzemień/rzemyk 'courroie, lanière, sangle', etc.

${ }^{42}$ Cette forme est indubitablement une transcription du russe кузнеи 'forgeron'.

${ }^{43}$ On peut y citer aussi des dérivés d'autres mots : tkać 'tisser', mielić 'moudre', warpać 'raccommoder', warzyć 'faire bouillir', etc.
} 
Barto|dziej 'celui qui fait des ruches (au début dans les troncs d'arbres)' (428)

Goli|broda 'celui qui rase la barbe' > barbier (13)

Goli|was 'celui qui rase la moustache' $(123)^{44}$

Koło $\mid$ dziej 'celui qui fait (fabrique) les charrettes' > charron (27898)

Pali|chleb ‘celui qui brûle le pain' > mauvais boulanger? (387)

Pali|maka 'celui qui brûle la farine' $>$ mauvais boulanger? $(527)^{45}$

Pieczy $\mid$ chlebek 'celui qui fait cuire le petit pain' > boulanger (88)

Piwo $\mid$ war 'celui qui brasse la bière' > brasseur, cervoisier (4198)

Soli|woda 'celui qui sale l'eau' (1062)

Warzy $\mid$ woda 'celui qui fait bouillir l'eau' (317)

Ces composés sont faiblement productifs quant à la suffixation. Parmi leurs dérivés, nous avons seulement : Bartodziejski, Bartodziejska, Palmaka, Palmączyński, Palmączyńska, Palmonczyński, Palmonczyńska, Soliwodzki, Soliwodzka, Soliwocki, Soliwocka, mais ils n'ont pas beaucoup de porteurs. Le nom Kołodziej et Piwowar sont une exception, étant les plus répandus, avec leurs nombreux dérivés : Kołodziej(cz)ak, Kołodziej(cz)uk, Kołodziejczycz, Kołodziejczyk, Kołodziejek, Kołodziejewski, Kołodziejewska, Kołodziejowicz, Kołodziejski, Kołodziejska et Piwowarek, Piwowarczuk, Piwowarczyk, Piwowarski, Piwowarska. De même, Stelmach, qui est le synonyme d'origine allemande de Kołodziej, a beaucoup de porteurs et beaucoup de formes dérivées. On peut donc en déduire que la production des chars était très répandue en Pologne. Probablement, la production de la bière était populaire aussi.

\section{II.3. NOMS ARTISANAUX ISSUS D'AUTRES LANGUES EN POLOGNE ${ }^{46}$}

- allemands : bütencere (moyen haut all.) 'tonnelier' : Bednar(z), Bednarzak, Bednarzowic, Bednar(cz)uk, Bednar(c)zyk, Bednarek, Bednarkiewicz, Bednarowicz, Bednarowski, Bednorz, Bednosz ${ }^{47}$; Schnitzer 'sculpteur en

\footnotetext{
${ }^{44}$ Le MC cite aussi 8 Goliwas, mais nous ne savons pas si c'est une autre notation de Goliwas ( $a$ au lieu de $a$ ), ou bien un autre nom qui pourrait se traduire 'celui qui vous rase'.

${ }^{45}$ D'autres variantes orthographiques : Palimaka (2), Palimonka (38).

${ }^{46}$ La Pologne ne possède qu'une langue régionale: le kachoube. Mais il y a d'importantes minorités historiques dont les langues sont considérées comme auxiliaires dans certaines régions : l'allemand, le lituanien et le bélorusse. Nous citons donc ici quelques exemples de noms issus de diverses langues historiquement liées à la Pologne, y compris le hongrois et le latin. Nous avons vérifié l'étymologie de ces noms dans : SEM, Rymut (1991, 1999, 2001).

${ }^{47}$ On peut y ajouter les noms passés par l'intermédiaire d'autres langues : hong. bodnár (emprunté au slave) > Bodnar, Bodner, Bodnarczuk, Bodnarek, Botnar, Botner; et ancien slave
} 
bois': Snycer(z), Snycerski; Stellmacher 'charron': Stelmach(er), Stal(l)mach, Stelmachowski, Stelmachów, Stelmaszak, Stelmaszek, Stelmasz(cz)yk, Stelmasz(cz)uk, Stelmaszewski, Stelmaszenko, Stelmaszyński, Stelmasi(a)k, Stelmaczonek, Stelmarczyk, Stalmacz(owski), Stalmaszczyk, S(z)tarmach, Starmaszak, Sztelmach, Sztalmach, etc. ${ }^{48}$

- latins : faber 'forgeron' : Faber(ka), Fab(i)erski, Fabirkiewicz, Fabrowicz, Fabrowski, Pabir, Pabirek, Vaberski; molo,-ere ou molendo,-are ${ }^{49}$ 'moudre" ${ }^{50}$ : Mole(n)da, Molęda, Molendowski, Molendys, Molendzki, Molęcki, Molędzki, Malenda, Malendowicz, Malendowski; molitor 'meunier' : Molitor(is), Molitorys ; pistor 'celui qui pile le grain dans un mortier; boulanger' : Pistor ; scriba 'copiste, scribe, greffier' : Skryba(nt), Skrybuch, Skrybunow, Skrybuś, Skryp(ka), Skrypko, Skrypoczka, Skrypczuk, Skrypuch, Skrypak, Skrypeć, Skrypski; sutor 'cordonnier': Sutor(czyk), Sutorkowicz, Sutorowicz, Sutorowski, Sutorski, Sutarz, Sutarczyk, Sutarski, Sutarzewicz, etc.

- hongrois : bognár 'charron' : Bognarz; kádár 'tonnelier' : Kadar ; lakatos 'serrurier' : Lakatosz ; molnár 'meunier': Molnar ; pintér 'tonnelier' : Pintér, Pinter(a); szabó 'couturier' (par le slovaque sabol 'couturier'): S(z)abała, Sabala(k), Sabalowicz, Sabalski; varga 'cordonnier' : Varga ${ }^{51}$, etc.

Finalement, on peut signaler que beaucoup de toponymes polonais viennent des noms de métiers des gens qui y habitaient en majorité, p.ex. Eagiewniki < tagiewnik 'tonnelier, celui qui faisait des récipients pour les liquides', Piekary < piekarz 'boulanger', Piwowary < piwowar 'brasseur, cervoisier', etc.

orient. боднарь qui, par métathèse, est devenu ukr. бондар et russe бондарь $>$ Bondar, Bondarczuk, Bondarek, Bondarenko, Bondarewicz, Bondarowicz, Bondaruk, etc.

${ }^{48}$ On peut y citer aussi : Gerber 'tanneur' > garbarz, Kannengießer 'ferblantier' > konwisarz, Tuchknabe 'garçon qui tissait les draps' > Knap 'tisseur', Kürs(ch)ner 'fourreur' > kuśnierz, Mälzer 'fabricant du malt' > Mielcarz, Müller 'meunier' > Mil(l)er, Riemer 'courroyeur' $>$ Rymarz, etc., ainsi que leurs nombreux dérivés.

${ }^{49}$ Cf. Gaffiot (1934: 988) qui cite une telle forme.

${ }^{50}$ Rymut $(1991,2001)$ donne ici l'étymon molendinator qui nous semble improbable, parce qu'une telle forme est introuvable dans diverses dictionnaires de latin, y compris le SŁŚ (qui cite pour le meunier: molenda, molendarius, molendinarius, molitor). Heureusement, le SEM en donne molenda. Cette forme pourrait être un participe du verbe molo,-ere ou bien venir d'une forme personnelle de molendo,-are. Le meunier français vient du lat. molinarius. Cependant, Bogusław Kreja (2001 : 52) dérive les noms de famille polonais Molenda et Molęda d'un ancien verbe pol. molić 'importuner, déranger'.

${ }^{51}$ Il n'est pas sûr si les formes similaires : Warga, Wargal(a), Wargas, Wargan, Wargawiak, Warganowski, dérivent du hongr. varga ou du pol. warga 'lèvre'. 


\section{II.4. STRUCTURE MORPHOLOGIQUE}

On observe une riche dérivation, surtout par les suffixes diminutifs, qui pouvaient désigner soit le fils de l'artisan soit son apprenti : le plus souvent $-i k /-(c z) y k /-(c z) e k$. Plus rarement -oś/-uś/-yś.

Il y a, bien sûr, des suffixes désignant l'agent, surtout : -arz/-orz/-erz/-nik. Plus rarement : -ar/-asz/-esz/-osz. Parfois, ces suffixes d'agent sont suivis de suffixes diminutifs.

Les suffixes -ak et -uk (celui-ci de dialectes de l'est ou d'origine ukrainienne) peuvent être considérés comme plutôt patronymiques.

La suffixation polonaise possède un riche répertoire, nourri de variantes dialectales, donc il est impossible de les énumérer tous ici (voir p.ex. Kreja 2001).

Excepté les noms adjectivaux en -ska, les matronymes sont difficilement discernables, parce que les formes terminées en - $a$ ne sont pas forcément féminines et pouvaient désigner aussi les hommes, p.ex. Cieśla 'charpentier' et quelques-uns de ses dérivés : Cieślica, Ciesielka, Ciesiołka, Ciesiótka, Ciesiutka ; ou Kowala, Kowara, Kowaczka, Kowalina, Krawania, Molenda, Molęda, Piekara, Piek(u)ta, Żarn(ot)a, Żarnowiczka, Żorna, ou bien Kalet(ka), Kalit(k)a, Kalitta, Lagwa, Rzeszotka, Cholewa, Watora. Le nom Kopyta peut même venir du pluriel du mot kopyto (dont le genre est neutre).

Certains métiers ne sont pas représentés parmi les noms de famille en Pologne, p.ex. kartownik 'cartier, fabricant de cartes à jouer', kottryniarz 'tapissier', ludwisarz 'fondeur de cloches', perukarz 'perruquier', płatnerz 'armurier, haubergier', szczytnik 'fabricant de boucles', etc. Peut-être, l'explication est-elle à chercher dans ce que nous avons déjà vu dans les noms français, p.ex. : certains de ces métiers pouvaient être sémantiquement associés à des notions plus larges comme forgeron, ou bien on préférait appeler de tels artisans avec les noms de leurs produits, parce que, par exemple, un mot était trop exotique et sophistiqué, comme koltryniarz qui vient de kottryna $<$ it. coltrina $(\text { sorte de tapisserie ornementale) })^{52}$, ou perukarz $<$ peruka $<$ fr. perruque $<$ it. parruca, etc. En fait, le temps de l'apparition de noms de certains métiers n'a pas de grande importance pour l'anthroponymie en Pologne, vu que les noms de famille ne se stabilisent qu' aux XVIII ${ }^{\mathrm{e}}$ et $\mathrm{XIX}^{\mathrm{e}} \mathrm{s}$.

\footnotetext{
${ }^{52}$ Selon le Treccani, ce mot est issu du croisement de cortina 'courtine' + coltre 'couverture' : «Cortina, come tenda nell'arredamento domestico (e anche, nell'uso ant., tipo di muratura di materiale laterizio)».
} 


\section{STATISTQUES ACTUELLES DES NOMS ARTISANAUX}

$\mathrm{Vu}$ les limites de l'article, nous n'avons pris que 50 noms de famille les plus populaires en France et en Pologne pour y voir la place des noms issus de l'artisanat.

\section{III.1. EN FRANCE}

Selon le registre de l'INSEE de 2008 : Martin, Bernard, Thomas, Petit, Robert, Richard, Durand, Dubois, Moreau, Laurent, Simon, Michel, Lefebvre, Leroy, Roux, David, Bertrand, Morel, Fournier, Girard, Bonnet, Dupont, Lambert, Fontaine, Rousseau, Vincent, Muller, Lefèvre, Faure, André, Mercier, Blanc, Guérin, Boyer, Garnier, Chevalier, François, Legrand, Gauthier, Garcia, Perrin, Robin, Clément, Morin, Nicolas, Henry, Roussel, Mathieu, Gautier, Masson.

Les noms issus de prénoms dominent, surtout ceux d'origine chrétienne et germanique. Il y a aussi des qualités physiques qui sont mises en relief.

Le premier nom issu d'un métier a la $13^{\mathrm{e}}$ place : c'est Lefebvre 'forgeron'. Ensuite, Fournier 'boulanger' a la $19^{\mathrm{e}}$ place. Plus bas, nous avons Muller 'meunier' d'origine allemande, ainsi que Lefèvre et Faure qui sont d'autres variantes du fr. Lefebvre, donc du lat. faber. Cette cinquantaine se clôt avec Masson 'maçon' d'origine germanique, mais l'aphérèse du prénom Thomasson (diminutif de Thomas) n'est pas exclue.

\section{III.2. EN POLOGNE}

Notre inventaire vient du recensement des noms portés par les hommes ${ }^{53}$ en Pologne, publié au mois de janvier 2018 (voir MC) : Nowak, Kowalski, Wiśniewski, Wójcik, Kowalczyk, Kamiński, Lewandowski, Zieliński, Woźniak, Szymański, Dąbrowski, Kozłowski, Jankowski, Mazur, Wojciechowski, Kwiatkowski, Krawczyk, Kaczmarek, Piotrowski, Grabowski, Zając, Król, Pawłowski, Michalski, Wróbel, Jabłoński, Wieczorek, Nowakowski, Majewski, Olszewski, Stępień, Jaworski, Dudek, Adamczyk, Malinowski, Pawlak, Górski, Sikora, Nowicki, Witkowski, Walczak, Baran, Rutkowski, Michalak, Szewczyk, Ostrowski, Tomaszewski, Zalewski, Wróblewski, Pietrzak.

\footnotetext{
${ }^{53}$ Les femmes ont un autre recensement qui est quand même assez similaire à celui des hommes.
} 
Ce sont les noms issus d'endroits qui dominent, ainsi que ceux issus de prénoms et d'animaux. Seulement 4 de cette cinquantaine, à savoir $8 \%$, viennent du métier artisanal : Kowalski qui tient la $2^{\mathrm{e}}$ place se compose de Kowal 'forgeron' + -ski (suffixe adjectival qui jouait ici un rôle descriptif, par conséquent il est devenu suffixe patronymique). Kowalczyk 'petit forgeron', Krawczyk 'petit couturier' et Szewczyk 'petit cordonnier' possèdent tous le suffixe diminutif $-y k$ qui pouvait désignait soit le fils de l'artisan, soit son apprenti. Il y a ici encore deux noms issus de métiers non artisanaux : Woźniak < woźny 'porteur de documents administratifs', puis 'huissier' et Kaczmarek < karczma 'auberge'. Eux aussi, ils possèdent des suffixes : -ak est typiquement patronymique et -ek est diminutif qui joue ici son rôle patronymique dans le sens 'fils/garçon/petit de l'aubergiste'.

\section{CONCLUSIONS}

Après avoir passé en revue les noms de famille artisanaux dans les deux pays, voici les différences et les ressemblances que l'on peut relever :

- En Pologne, les noms de famille ont une dérivation beaucoup plus riche et ont des variantes plus nombreuses que ceux en France.

- Les suffixes d'agent dominent en France, alors qu'en Pologne, ce sont les diminutifs qui sont les plus nombreux.

- En France, les noms ont moins de syllabes, tandis qu'en Pologne, ils peuvent être longs et cumuler même deux ou trois suffixes. Voire les noms composés polonais peuvent subir la suffixation (ce qui ne se voit pas dans les noms composés français). Par contre, dans certains noms en France, on voit l'article défini qui accompagne le nom, voire souvent il est agglutiné.

- Les noms composés sont plus rares en Pologne qu'en France.

- Dans les deux pays, on voit une grande fréquence du nom d'un produit ou d'un objet du travail en tant que base du nom de métier (en France, cela s'observe surtout quand le nom de métier est long).

- En Pologne on trouve aussi une partie d'un produit comme base du nom (p.ex. une partie de la chaussure). En France, cela n'apparaît pas si clairement.

- Dans les deux pays, l'action faite au travail sert de base aussi, mais plus rarement.

- L'endroit du travail apparaît rarement dans les noms, mais, curieusement, dans les deux pays, c'est le moulin et la forge qui dominent.

- Les matronymes sont rares dans les deux pays. 
- Les noms de famille français sont souvent plus difficilement reconnaissables quant à leur étymologie que les noms polonais dont la plupart se laissent assez facilement discerner à un locuteur moyen. Cela est dû, sans doute, à une évolution phonétique plus dynamique du français et de ses dialectes, y compris les divergences entre les domaines de la langue d'oc et de la langue d'oïl. En Pologne, les différences dialectales ne sont pas si profondes. En outre, puisque les noms polonais sont, d'habitude, plus longs, ils sont plus transparents.

Bien sûr, dans d'autres pays, les noms de métiers sont présents dans les noms de famille aussi. Certains sont devenus très populaires, comme dans les pays germaniques ${ }^{54}$, p.ex. : en Grande Bretagne et en Allemagne : Smith $(\mathrm{GB})=$ Schmidt, Schmitt, Schmied $(\mathrm{A})$ 'forgeron'; Carpenter $(\mathrm{GB})=$ Zimmermann (A) 'charpentier'; Taylor (GB) = Schneider (A) 'couturier, tailleur'; Cooper $(\mathrm{GB})=$ Binder, Bender, Fassbinder, Fassbender, Böttcher, Boettcher 'tonnelier', etc.

Dans les pays slaves, de tels noms sont assez populaires aussi, ce que nous avons vu dans les exemples polonais ci-dessus. Parmi d'autres, p.ex. serbo-croate Коvас̌/Ковач, russe Кузнеи 'forgeron'; s.-c. Tesar/Tecap, russe Плотник 'charpentier' ; s.-c. Krojač/Кројачч ${ }^{55}$, russe Портной 'couturier, tailleur'; s.-c. Bačvar/Бачвар et Kačar/Качар, russe Бондарь et Бочар 'tonnelier', etc. Le plus souvent, ils sont munis de divers suffixes.

Cependant en Roumanie qui est un pays roman très slavisé, il y a assez peu de noms de ce type, mais on en trouve, le plus souvent avec le suffixe $-u$ : fierar 'forgeron' > Fieraru, dulgher 'charpentier' > Dulgheru, croitor 'couturier, tailleur' > Croitoru, dogar / butnar 'tonnelier' > Dogaru/Butnaru.

Dans le sud de l'Europe, surtout dans d'autres pays romans, les noms de métiers sont encore moins populaires parmi les noms de famille. Le forgeron est le seul à avoir une assez grande fréquence, p.ex. en Italie, le nom Ferrari ( $<$ lat. [faber] ferrarius 'forgeron, ferronnier') a la $3^{\mathrm{e}}$ place (après Rossi et Marchetti $)^{57}$ et a beaucoup d'autres formes (p.ex. Ferrero, Ferrelli, Ferrarello, Ferraretto, Ferrarini, etc.), tandis qu'en Espagne Herrero, Herrara et au Portugal Ferreiro, Ferreira sont plus rares.

\footnotetext{
${ }^{54}$ Sauf les pays nordiques où ce sont les prénoms qui dominent, surtout dans les patronymes terminés par -son 'fils de' (et -dóttir 'fille de' en Islande). Dans les noms de famille scandinaves, on voit souvent aussi la topographie et les éléments de la nature. Il en va de même avec la Finlande.

${ }_{55}^{55}$ Dans les noms de famille, le plus souvent avec - $a-$ : Krajač/Kpajaч.

${ }^{56}$ Celui-ci comme nom de famille est presque toujours avec la terminaison -ov : Бочаров.

${ }^{57}$ Cf. https://www.mappadeicognomi.it/classifica_cognomi_piu_diffusi.php (consulté en mars 2019).
} 


\section{BIBLIOGRAPHIE}

Abalain Hervé, 2004, Pleins feux sur la langue bretonne, Spézet, Coop Breizh.

Alonso Javier, 2018, La Heráldica de Javier Alonso: https://heraldicajavieralonso.jimdo.com/ significado-apellidos-vascos/ (consulté en mars 2019).

Carrez Henri, 1927, "L'origine des noms de famille dans la Lorraine de langue française », Mémoires de l'Académie nationale de Metz, 1926, 245-258.

Géraud Hercule, 1837, Paris sous Philippe-le-Bel : d'après des documents originaux et notamment d'après un manuscrit contenant le rôle de la taille imposée sur les habitants de Paris en 1292, Paris, Imprimerie de Crapelet.

Kaleta Zofia, 1998, Nazwisko w kulturze polskiej, Warszawa, Slawistyczny Ośrodek Wydawniczy.

Kreja Bogusław, 2001, Słowotwórstwo polskich nazwisk : struktury sufiksalne, Kraków, DWN.

Lebel Paul, 1959, Les noms de personnes en France, Paris, Presses Universitaires de France.

Malec Maria, 2008, « Dawne nazwy zawodów typu kołodziej, piwowar jako baza leksykalna polskich nazwisk », Acta Onomastica, 49, 227-235.

Michelena Luis, 1973, Apellidos vascos, San Sebastian, Txertoa.

Rudnicka-Fira Elżbieta, 2004, Antroponimia Krakowa od XVI do XVIII wieku : proces ksztaltowania się nazwiska, Katowice, Wydawnictwo Uniwersytetu Śląskiego.

\section{SOURCES}

$\mathrm{ALF}=$ Atlas linguistique de la France, disponible sur le site: http://lig-tdcge.imag.fr/ cartodialect3/ (consulté en mars 2019).

Dauzat Albert, 1949, Traité d'anthroponymie française : les noms de famille de France, Paris, Payot.

Dauzat Albert, 1951, Dictionnaire étymologique des noms de famille et prénoms de France, Paris, Larousse.

EAV = Etimología de Apellidos Vascos : https://martzelmarchant.wordpress.com/ (consulté en mars 2019).

FEW = Wartburg Walther von (1922-1967), Französisches Etymologisches Wörterbuch, disponible sur le site https://apps.atilf.fr/lecteurFEW/ (consulté en mars 2019).

Franklin Alfred, 1906, Dictionnaire historique des arts, métiers et professions exercés dans Paris depuis le treizième siècle, Paris \& Leipzig, H. Welter.

Gaffiot Félix, 1934, Dictionnaire latin-français, Paris, Hachette.

Godefroy = Godefroy Frédéric (1891-1902), Dictionnaire de l'ancienne langue française et de tous ses dialectes $d u I X^{e}$ au $X V^{e}$ siècle, Paris, F. Vieweg, 10 tomes.

INSEE = Fichier des noms sur le site de l'Institut national de la statistique et des études économiques : https://www.insee.fr/fr/statistiques/3536630 (consulté en mars 2019).

Kremer Dieter (coord.), 1997, Dictionnaire historique de l'anthroponymie romane (PatRom), Berlin \& Boston, De Gruyter.

MC = Statystyka nazwisk - Polska $i$ województwa - styczeń 2018 - Kobiety \& Mężczyźni : sur le site du Ministerstwo Cyfryzacji (Ministère de la Numérisation) de Pologne : https://www.gov.pl /web/cyfryzacja/dodatkowe-materialy (consulté en mars 2019).

Rymut Kazimierz, 1991, Nazwiska Polaków, Wrocław, Ossolineum. 
Rymut Kazimierz, 1999, Nazwiska Polaków : słownik historyczno-etymologiczny, t. 1, A-K, Kraków, Wydawnictwo Instytutu Języka Polskiego PAN.

Rymut Kazimierz, 2001, Nazwiska Polaków : słownik historyczno-etymologiczny, t. 2, L-Ż, Kraków, Wydawnictwo Naukowe DWN.

SEM = Stownik etymologiczno-motywacyjny staropolskich nazw osobowych, vol. 1, Odapelatywne nazwy osobowe, Aleksandra Cieślikowa, Maria Malec, Kazimierz Rymut, Kraków, DWN, 2000.

SŁŚ = Stownik Łaciny Średniowiecznej w Polsce, Kraków, Instytut Języka Polskiego PAN, disponible sur le site : http://scriptores.pl/elexicon/ (consulté en mars 2019).

TLFi = Trésor de la langue française informatisé, disponible sur le site : http://atilf.atilf.fr/tlfi.htm (consulté en mars 2019).

Treccani = Enciclopedia Italiana di Scienze, Lettere ed Arti dall'Istituto Giovanni Treccani : www.treccani.it/vocabolario (consulté en mars 2019).

\section{NOMS DE FAMILLE ISSUS DE L'ARTISANAT EN FRANCE ET EN POLOGNE}

\section{Résumé}

L'article analyse les noms de famille issus de noms artisanaux en France et en Pologne. Il présente leurs origines (y compris des influences étrangères), types et formation de mots. On peut voir, entre autres, que les noms de famille français sont plus courts, mais ont beaucoup de variantes dialectales, tandis que les noms de famille polonais sont plus longs et ont une dérivation plus riche. L'article montre aussi la position de tels noms de famille dans les statistiques démographiques des deux pays : le forgeron comme étymon s'avère le plus populaire. Dans la top cinquantaine on peut trouver encore en France : fournier (boulanger), meunier et maçon ; tandis qu'en Pologne : couturier et cordonnier.

Mots-clés : noms de famille; patronymes; artisanat; artisan.

\section{NAZWISKA ODRZEMIEŚLNICZE WE FRANCJI I W POLSCE}

\section{Streszczenie}

Artykuł analizuje nazwiska pochodzące od nazw rzemieślniczych we Francji i w Polsce. Przedstawia ich pochodzenie (w tym wpływy obce), typy i słowotwórstwo. Można stwierdzić między innymi, że nazwiska francuskie są krótsze, ale mają wiele wariantów dialektalnych, podczas gdy nazwiska polskie są dłuższe i mają bardziej bogatą derywację. Artykuł podaje także ranking takich nazwisk w statystykach demograficznych obu krajów: kowal jako etymon okazuje się najbardziej popularny. W pierwszej pięćdziesiątce we Francji są jeszcze: piekarz, młynarz i murarz; zaś w Polsce: krawiec i szewc.

Słowa kluczowe: nazwiska; patronimy; rzemiosło; rzemieślnik. 